\title{
Numerical Solutions of Population Balance Equations within Liquid/Gas-Liquid Flow Simulations
}

\author{
Evren Bayraktar*, Otto Mierka and Stefan Turek \\ Institute of Applied Mathematics (LS III), TU Dortmund \\ Vogelpothsweg 87, D-44227, Dortmund, Germany. \\ E-mail: evren.bayraktar@mathematik.tu-dortmund.de
}

\begin{abstract}
Numerical solvers based on population balance equations (PBE) coupled with flow equations are a promising approach to simulate liquid/gas-liquid dispersed flows which are very commonly observed in the nature and in industrial processes. Our aim is to discuss the challenges for the numerical solution of the coupled equation systems and to present detailed numerical recipes whose main ingredients are the method of classes, positivity-preserving linearization and the high-order FEM-AFC schemes, additional to our in-house flow solver software package FEATFLOW. We study liquid-liquid flows through a static mixer and dispersed phase systems in a flat bubble column with the accordingly developed computational tool. The suggested recipes were validated by comparing the numerical results against experimental data so that we present a novel approach to the numerical simulation of bubbly flows at moderate gas holdups, which involves highly accurate numerical techniques and is computationally efficient and robust.
\end{abstract}

Keywords: Population balance equations, Computational fluid dynamics, Bubble columns, Dispersed flows, Numerical methods.

\section{Introduction}

Dispersed two-phase flows are one of the most common flow types observed both in the nature and in industrial processes. It can be the motion of clouds, the movement of a smoke plume, or the dynamics of fluids in processes which are interesting to food, petroleum, chemical, pharmaceutical and many other industries; they are all governed by the same 
physical laws. Even though the dispersed phase systems are encountered so often in daily life and in the industrial processes worth billions of Euros, our understanding of the physical laws governing the dispersed phase systems is still quite limited and indeed, it is not due to lack of interest in this research field but "simply" due to the fact that the physical phenomena are highly complex and tangled.

In practical applications, a single droplet size model, as reported by numerous researchers [1, 2], cannot properly describe the interfacial interactions between the phases; therefore, population balance equations (PBE) are employed to describe the evolution of the secondary phase in the internal coordinate(s) and in time. The first efforts which can be related to concepts of population balances appeared at chemical engineering applications in the 1960s, and there has been since then an intensive ongoing research in this field by researchers of many different backgrounds.

PBE are integro-partial differential equations, exhibit analytical solutions for very few cases only which are mostly not able to describe the applications in practice, and the use of appropriate numerical techniques is unavoidable in order to deal with practical problems. There are several numerical methods satisfying the necessary requirements with respect to robustness and realizability: the quadrature method of moments (QMOM) [3, 4], the direct quadrature method of moments (DQMM) [5], parallel parent and daughter classes (PPDC) [6] and the method of classes (MC) [7, 8]. Among these techniques, a moment based method PPDC and a sectional method MC are in our focus.

The dynamics of bubble columns has been a very interesting topic to researchers in the field of CFD for the last several decades and many different methods have been developed. Numerical simulation of flow fields in bubble columns is possible by adopting the EulerLagrange or Euler-Euler approaches. For practical reasons, e.g., high numerical efforts and computational costs which are related to tracking and calculating the motion of each bubble individually in the flow field, the former method is restricted to be applied on lean dispersions only while the later method requires less effort in both numerics and computation. Nevertheless, both of the methods lead to the same results if the problems are handled with adequate computational effort as it has been reported by Sokolichin et al. [9]. We followed Sokolichin et al. and adopted the Euler-Euler approach to describe the two-phase system. 
In the Euler-Euler approach, one should employ one of the following models: mixture model, two- and multi-fluid model; and solving the continuity equation for two-/multi-fluid models is very troublesome because the continuity has to be satisfied with the numerical solutions of the weakly coupled phase velocities, that exhibit a great numerical challenge for Navier-Stokes solvers [10], especially for segregated ones. The choice of the fluid model is not independent of how the PBE are coupled to the flow part. One-way coupled CFD-PBE models can not comprehensively describe the dynamics of bubble columns. Therefore, a twoway coupled CFD-PBE model is required and beginning from the employed discretization method for the internal coordinate, each adopted approach influences the decision on the fluid model.

The PBE do not need to be solved on the same computational grid (in space and in time) with the flow equations due to excessive computational costs. The first attempt to reduce the required computational afford may be the compartment method [11] which is based on the idea of dividing the computational domain into very large units, namely compartments, and solving the PBE in each compartments with the averaged values of fluid variables. This method can be employed to simulate the liquid-liquid dispersions in stirred tanks for which certain regions have quasi-steady flow behavior whereas for the applications like bubble columns, i.e., when the interaction of the phases should be considered in a twoway direction, the compartment method is not good anymore and the PBE must be solved for the flow problem's number of degrees of freedom, considering the number of required compartments is on the order of 10 , this requires a great computational effort. Moreover, now the non-linear scalar transport problem needs to be solved for the dispersed phase. And, all these add up to an enormous computational effort hence the use of efficient numerical techniques are of predominant importance.

A comprehensive PBE-CFD model involves a two- or multi-fluid model for the momentum balance [12]. If all the bubbles are assumed to share the same velocity field, only one momentum equation is solved for the dispersed phase, that is very common in case of a moment based method to be adopted to solve PBE, it is also used with MC [13-14]. Nevertheless, within both methods there have to be solved additional convection-diffusionreaction (CDR) problems. These problems are either transport of the low-order moments or certain property (properties) of classes (multi-dimensional PBE) and the number scales with the required number of moments or the employed number of classes. Nevertheless, multi-fluid models can be more preferable when MC is chosen; then, a separate momentum equation is 
solved for each class. There are three main difficulties which are inherited from multi-fluid models independent of PBE: (i) how to satisfy the continuity equation, (ii) excessive computational costs of the matrix assembly, (iii) determining the interphase forces. Moreover, not only solving a large number of transport problems is challenging, another problem is to determine the appropriate advection velocity, especially for the moment based methods [15, 16]. The size of the second difficulty can be understood better, if it is considered that each class is a separate phase, for each one the discrete convection operator has to be assembled, and the cost of assembling the convection matrix is much more than solving the associated linear equation with the CDR problem.

The third difficulty should be considered in detail since it is the coupling term between the momentum equations. There are three main interphase forces, the virtual mass force ( $\left.f_{V M}\right)$, the lift force $\left(f_{L}\right)$ and the drag force $\left(f_{D}\right)$, and in our case we only work on the drag force. The drag force seems to be the only force which has significant influence and clear definition even tough there are various definitions of the drag coefficient. On the other hand, $f_{V M}$ is not influential and can be freely neglected for the sake of reducing the computational cost, and $f_{L}$ has extremely deviating formulations; moreover, it is frequently abused for fitting numerical results to experimental data $[2,17]$.

We preferred a simplified model and tackled the problem within the Euler-Euler approach. The dispersed system is considered as a mixture and a single momentum equation is solved for the fluid in the framework of the mixture model and the algebraic slip relation is employed to recover the gas phase velocity. Discretized PBE with MC are coupled to turbulent flow equations in a two-way fashion. While the hydrodynamic variables are coupling the PBE equation to flow solutions, the formulation of the drag force requires the solution of PBE. The turbulence is modeled with the standard $k-\varepsilon$ turbulence model and buoyancy induced turbulence. Buoyancy is handled with a relaxed Boussinesq approximation. Then, the continuity equation is solved with the constant density assumption and the local gas holdup is calculated regarding the ideal gas law. We assume that all the bubbles share the same velocity field and this velocity is obtained by using the Sauter mean diameter of the population within the algebraic slip relation. 


\section{Mathematical model and numerical approaches}

The developed complete mathematical model is based on an Euler-Euler approach and consists of three parts: (i) the hydrodynamic core, a model to solve the "incompressible" Navier-Stokes equations; (ii) the simplified two-fluid model based on an analog of Boussinesq approximation for natural convection problems; (iii) PBE to describe the size distribution of the dispersed phase, the schematic view of how these models are connected is given in Figure 1.

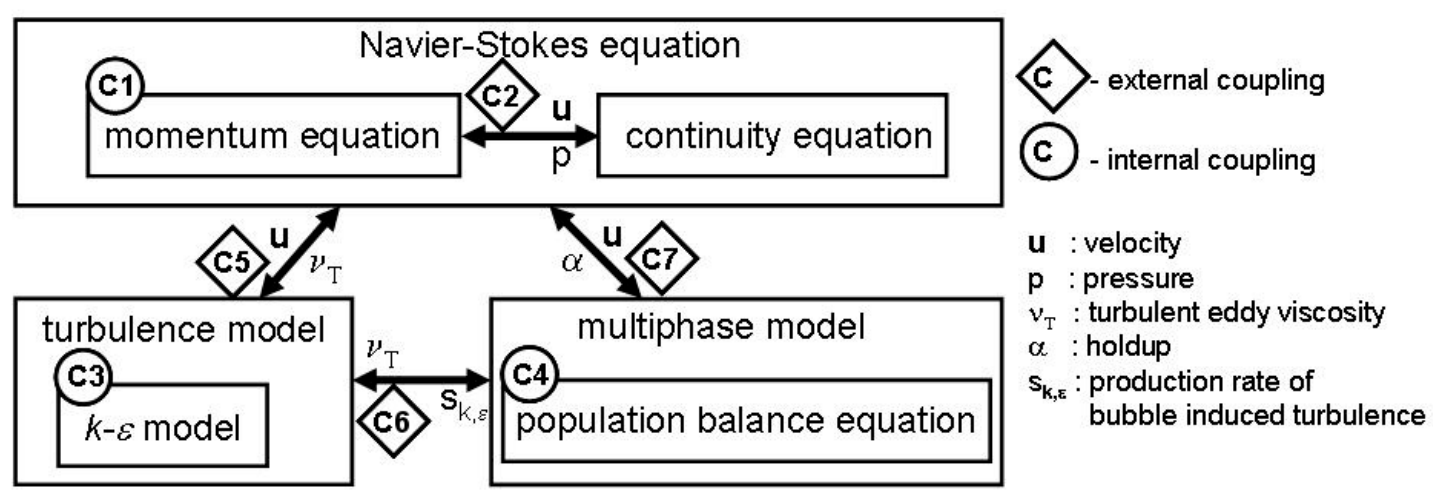

Figure 1: Sketch of the coupling effects inside the complete model.

The second part restricts the model to be valid for bubbly flows with moderate gas holdups (up to 10\%). Under such circumstances, “incompressible” Navier-Stokes equations are enriched with an extra buoyancy force term, by what the gas-liquid mixture is characterized as a weakly compressible fluid. Consequently, the dispersed phase system can be considered as space-sharing interpenetrating continua. The major advantage of this approach is that the computational cost is independent of the number of bubbles to be simulated.

Replacing the effective density $\tilde{\rho}_{L}$ by the liquid density $\rho_{L}$ except for the gravitational force leads to the Navier-Stokes equations for the liquid phase as described in the following:

$$
\begin{aligned}
& \frac{\partial u_{L}}{\partial t}+u_{L} \cdot \nabla u_{L}=-\nabla p+\nabla \cdot\left(v_{T} D\left(u_{L}\right)\right)-\alpha g, \\
& \nabla \cdot u_{L}=0, \quad p=\frac{p-p_{a t m}}{\rho_{L}}+g \cdot x, \quad \alpha=\frac{\tilde{\rho}_{G}}{\rho_{G}}
\end{aligned}
$$


where $D(u)=\nabla u+\nabla u^{T}$ and the effective viscosity $v_{T}=C_{\mu} \frac{k^{2}}{\varepsilon}$ is a function of the turbulent kinetic energy $k$ and its dissipation rate $\varepsilon$. The continuity equation of the gas phase and the evolution of the turbulent quantities are described by the following equations,

$$
\begin{gathered}
\frac{\partial \tilde{\rho}_{G}}{\partial t}+\nabla \cdot\left(\tilde{\rho}_{G} u_{G}\right)=0, \\
\frac{\partial k}{\partial t}+\nabla \cdot\left(k u_{L}-\frac{v_{T}}{\sigma_{k}} \nabla k\right)=P_{k}+S_{k}-\varepsilon, \\
\frac{\partial \varepsilon}{\partial t}+\nabla \cdot\left(\varepsilon u_{L}-\frac{v_{T}}{\sigma_{k}} \nabla \varepsilon\right)=\frac{\varepsilon}{k}\left(C_{1} P_{k}+C_{\varepsilon} S_{k}-C_{2} \varepsilon\right),
\end{gathered}
$$

where the production terms $P_{k}=\frac{v_{T}}{2}\left|\nabla u+\nabla u^{T}\right|^{2}$ and $S_{k}=-C_{k} \alpha \nabla p \cdot u_{\text {slip }}$ are due to the shear and bubble-induced turbulence (BIT), respectively. The involved constants $C_{\mu}=0.09$, $C_{1}=1.44, C_{2}=1.92, \sigma_{k}=1.0, \sigma_{\varepsilon}=1.3$ for the standard $k-\varepsilon$ model are known with high precision, whereas the BIT parameters $C_{k} \in[0.01,1]$ and $C_{\varepsilon} \in[1,1.92]$ are highly problemdependent.

After decomposition of the interphase force term responsible for momentum exchange between the present two phases and using the assumptions introduced by Sokolichin et al. $[17,19]$, the momentum balance of the gas phase reduces to:

$$
0=-\alpha \nabla p-\alpha C_{W} u_{\text {slip }}, \quad C_{W}=C_{D} \frac{3}{8} \frac{\rho_{L}}{r}\left|u_{\text {slip }}\right|
$$

and the gas velocity (2.6) can be given with the following definitions of the slip velocity (2.7) and the drift velocity (2.8) as,

$$
\begin{gathered}
u_{G}=u_{L}+u_{\text {slip }}+u_{\text {drift }}, \\
u_{\text {slip }}=\frac{-1}{C_{D} \frac{3}{8} \frac{\rho_{L}}{r}\left|u_{\text {slip }}\right|} \nabla p, \quad \text { where } C_{D}=0.666, \\
u_{\text {drift }}=\frac{-v_{T}}{\sigma_{G}} \frac{\nabla \alpha}{\alpha} .
\end{gathered}
$$

We should remark that $u_{\text {drift }}$ is not calculated explicitly but is accounted for the dispersion. $C_{D}$ (2.7) is adopted from the study by Bannari et al. [13] in which several other formulations of $C_{D}$ are comprehensively discussed; even though the chosen value of $C_{D}$ does not provide the most accurate results, it has the advantage that it yields to a linear 
formulation of $u_{\text {slip }}$ (2.7) after some manipulation of the equation (2.7). With other formulations of $C_{D}$ which involve $u_{\text {slip }}$, additional nonlinearity arises; those demand even more computational efforts. Since our focus is mostly on investigating the couplings between PBE and equations of fluid dynamics, we have chosen the constant drag coefficient in (2.7) which has proven to yield sufficiently accurate results [20]. The implementation is realized such that in further studies, any other desired definition of $C_{D}$ can be easily adopted with an explicit treatment without changing any other part of the developed computer program but the respected subroutine.

The term $r$ (radii of bubbles) which appears in the definition of the $C_{W}$ (2.5) linearization parameter in the formulation of $u_{\text {slip }}$ couples the system to the PBE. One common practice is to adopt a certain value for $r$ and having a model without PBE; however, this is not a general solution, even though it may lead to acceptable results for specific cases. Moreover, even it can be considered that $u_{\text {slip }}$ has a certain value depending on the initial and boundary conditions, so that the model is simplified. In general, $u_{\text {slip }}$ has the opposite direction of the gradient of the pressure, and the square of its magnitude is proportional to the magnitude of the pressure gradient unless $C_{D}$ is a function of $u_{\text {slip }}$. The adopted algebraic slip relation can easily provide a specific advection velocity for each class; nevertheless, this would lead to a stronger coupling of PBE and the flow part. Then, PBE had to be solved in the same outer loop with the CFD part, see Figure 2, and this would result in much higher computational costs since it would require solving the PBE several times in one time step. Assuming that the local variation of bubble sizes is not significantly changing during one sufficiently small time step, we say that bubbles forming one ensemble can be advected with a certain local advection velocity which we obtain by using the Sauter mean diameter ( $d_{32}=\sum N_{i} d_{i}^{3} / \sum N_{i} d_{i}^{2}$ ), that accurately represents bubbles' sizes in an ensemble. So that, by advecting all the bubble classes within a common velocity field we do not only solve PBE and equations of the fluid dynamics in a segregated fashion but we avoid assembling a discrete convective operator for each class. If we take into account that assembly of discrete transport operators takes much more time than solving the associated linear problem, the extend of the gain in computational effort will be more obvious.

We wish to investigate the effect of size distribution of the bubbles by obtaining a representative size of bubble ensembles, which is the Sauter mean diameter; consequently, we 
incorporate our fluid model with PBE which can be given as,

$$
\begin{aligned}
& \frac{\partial f}{\partial t}+\nabla \cdot\left(u_{G} f\right)-\nabla \cdot\left(\frac{v_{T}}{\sigma_{T}} \nabla f\right)=\int_{v}^{\infty} r^{B}(v, \tilde{v}) f(\tilde{v}) d \tilde{v}-\frac{f(\tilde{v})}{v} \int_{0}^{v} \tilde{v} r^{B}(\tilde{v}, v) d \tilde{v} \\
& \quad+\frac{1}{2} \int_{0}^{\infty} r^{C}(\tilde{v}, v-\tilde{v}) f(\tilde{v}) f(v-\tilde{v}) d \tilde{v}-f(v) \int_{0}^{\infty} r^{C}(\tilde{v}, v) f(\tilde{v}) d \tilde{v}
\end{aligned}
$$

Equation (2.9) is an integro-partial differential equation which describes how the dispersed phase evolves in time, space and size coordinate, in its discrete form it exhibits strong internal and external couplings (see c4, c6 and c7 in Figure 1). The external couplings are due to the turbulent diffusivity and the velocity which are given with $v_{T} / \sigma_{T}$ and $u_{G}$. The internal couplings will be more apparent when the discrete counterpart of (2.9) is given. One important remark here is about the treatment of the diffusivity; as the air bubbles are accepted to be non-diffusive in water, there should be no diffusion in our model; however, since the fluid dynamics is described with RANS model, the turbulent viscosity arises which is responsible for the diffusive term in (2.9). Hence, the system is highly convection dominant, high-resolution positivity preserving schemes are required for accuracy purposes and we employ an implicit high order finite elements method with TVD type limiters with positivity preserving linearization so that the overshoots and undershoots are avoided, for details see [2, 21-23].

The discrete counterpart of the population balance equation (2.9) is carried out with the fixed pivot technique in method of classes. The fixed pivot volume of the classes is initialized by specifying the volume of the smallest "resolved" class $v_{\min }$ and the discretization factor $q$, such that

$$
v_{i}=v_{\min } q^{i-1} \quad \text { with } \quad i=1,2, \ldots n
$$

where $n$ is the number of classes. The class width $\Delta v_{i}$ is defined by the difference of the upper $v_{i}^{U}$ and lower $v_{i}^{L}$ limits of the given class $i$ :

$$
\Delta v_{i}=v_{i}^{U}-v_{i}^{L} \quad \text { with } \quad v_{i}^{U}=v_{i+1}^{L} \text { and } \quad v_{i-1}^{U}=v_{i}^{L} .
$$

The limits are fixed and initialized such that in the case of $q=2$ the pivot volume $v_{i}$ is centered in the class

$$
v_{i}^{U}=v_{i}+\frac{1}{3}\left(v_{i+1}-v_{i}\right), \quad v_{i}^{L}=v_{i}-\frac{2}{3}\left(v_{i}-v_{i-1}\right) .
$$

Following equation (2.12), the discrete internal coordinate can be sketched as in Figure 2. 


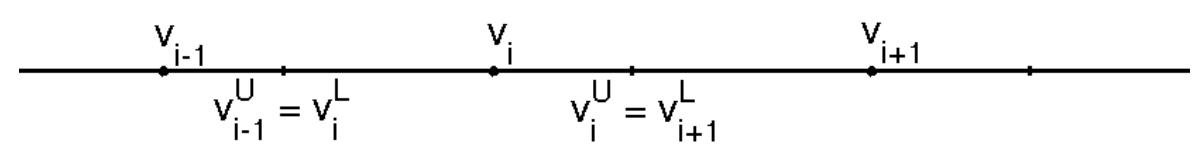

Figure 2: Discrete internal coordinate with MC.

The discretized transport equation (2.9) of the $i$-th class' number density probability, $f_{i}$, in a discretized form reads

$$
\begin{gathered}
\frac{\partial f_{i}}{\partial t}+u_{g} \cdot \nabla f_{i}+\nabla \cdot\left(\frac{v_{T}}{\sigma_{T}} \nabla f_{i}\right)=\sum_{j=i}^{n} r_{i, j}^{B} f_{j} \Delta v_{j}-\frac{f_{i}}{v_{i}} \sum_{j=1}^{i} v_{j} r_{j, i}^{B} \Delta v_{j} \\
\quad+\frac{1}{2} \sum_{j=1}^{i} r_{j, k}^{C} f_{j} f_{k} \Delta v_{j}-f_{i} \sum_{j=1}^{n} r_{j, i}^{C} f_{j} \Delta v_{j} \quad \text { for } i=1,2, \ldots n,
\end{gathered}
$$

where $r_{i, j}^{B}$ and $r_{j, k}^{C}$ are the breakage and coalescence kernels, respectively, which will close PBE (2.13). In the literature, there are many published studies on modeling of breakage and coalescence kernels, Chen et al. presents a detailed comparison of several important kernels [24], and we adopted the coalescence and breakage kernels from Lehr et al. [25], that we conclude as more favorable in our previous study [21]. The main reasons that we have chosen these pair of kernels are: the convergence behavior with the number of parent classes, their accuracy and the applicability in a wide range of operating conditions, a detailed study is given in [21].

A last point worth to be discussed is the implementation of boundary conditions which is not a trivial subject; we follow the prescriptions by Kuzmin et al. [2]. At the inflow boundary all velocity components and turbulent quantities, $k$ and $\varepsilon$, are prescribed. At the outlet 'donothing' boundary condition is employed which states that all the normal gradients of all variables vanish. The walls are modeled with the free-slip boundary condition which requires the computation of walls' normal which can be cumbersome in case of the walls are not aligned with the chosen coordinate system. The gas inflow rate can be incorporated as a source into the gas phase continuity equation, and bubbles are introduced at the inflow having a size of $1 \mathrm{~mm}$ which corresponds to the median class $4^{\text {th }} / 6^{\text {th }}$ class for $7 / 11$ classes. For the further implementation details of boundary and initial conditions, and the wall functions, we encourage the reader to refer to the following studies [2, 30, 31].

Hereby, we have completed our discussion on the mathematical model and the numerical approaches and now we can present our computational recipe which explains step by step our approach to the solution of the described mathematical model within the time 
step $t^{n}$ towards $t^{n+1}$.

1. Solve the Navier-Stokes equations.

2. Obtain the gas velocity.

3. Solve the transport equation for the effective gas density/ holdup $\alpha$.

4. Solve the coupled equations of the $k-\varepsilon$ model.

5. Check the convergence criteria for the outer-loop (1-4) for the solution of the CFD equations,

5.1. YES: pass the necessary values to population balance model and progress to solve PBE, go to 6 .

5.2. NO: go on with the next iteration in the outer-loop, go to 1 .

6. Solve internally the coupled PBE in the outer-loop

6.1. Calculate the sink and source terms for PBE.

6.2. Solve the transport problem for each class.

7. Check the convergence criteria for the outer-loop (6),

7.1. YES: calculate $d_{32}$ of the population and pass it to the fluid dynamics part, 2, for $t^{n+1}$.

7.2. NO: go on with the next iteration in the outer-loop, go to 6.1 .

Our implementation strategies of the internal and external couplings, which are sketched in Figure 1, within our computational recipes give rise to the nested iterations which are shown in Figure 3 which is important to see how the couplings are handled and how our recipes are implemented within the nested iteration strategy; moreover, it gives an idea about the computational demand of our accordingly developed computational tool.

As a summary, our computational approach shall be explained with all details for one time step: At time step $t^{n}$ one should first solve the Navier-Stokes equations which are modified with the Boussinesq approximation, (2.1)-(2.2). To solve these equations the employed numerical methods are: FEM on unstructured grid, Pressure-Schur-Complement approach which yields to the c2 external coupling, the loop with the $l$ indices (loop $l$ ). Since the Burgers equations for the velocity components are solved in a decoupled fashion, which resulted in the c1 internal coupling. The obtained linear equations for the velocity and pressure unknowns are solved with an efficient geometrical multigrid solver in a defect correction loop, $\operatorname{loop}_{m}$. Then, the transport equation for the total gas holdup, $\alpha$, is solved with the obtained gas velocity being computed with $d_{32}$ from the PB part. Since this equation and 


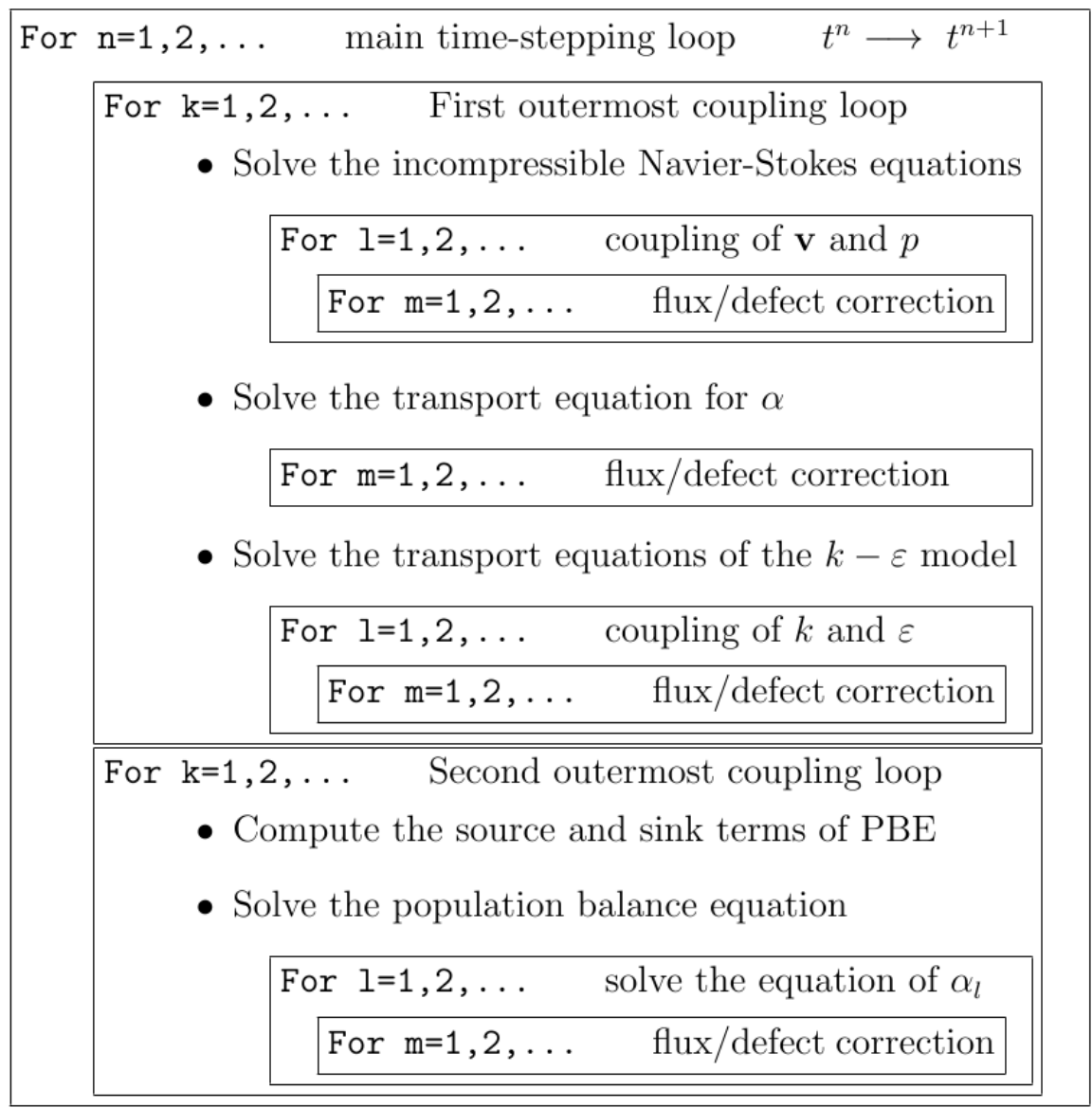

Figure 3: Developed computational algorithm consisting of nested iteration loops.

the other scalar transport equations in our model are convection dominated, an SOR-solver on the finest computational grid is employed with an Algebraic Flux Correction (AFC) scheme additional to the positivity-preserving linearization in order to ensure valid non-negative solutions. Then the coupled system of $k-\varepsilon$ equations is analogously solved in $\operatorname{loop}_{l}$. So far, the steps 1-4 have been realized. Since all the involved equations are strongly coupled, steps 1-4 have to be performed in the first outermost coupling loop. We consider that the coupling between PBE and CFD is weak, i.e., we assume that the size distribution of the bubbles has been changing negligibly while we are computing the fluid dynamics; so that, the slip velocity remains constant in the first outermost coupling and we can solve PBE later. This assumption reduces the computational cost considerably so that we are able to numerically simulate our problems with the developed sequential computational tool. Once we obtained the converged solution of the CFD part, we progress with the solution of the PBE. First the sink and source terms are computed with MC, step 6.1, then the related terms are incorporated into the transport equation of $\alpha_{l}$, which is solved analogously to the previous scalar transport equations. Since the values of the classes strongly depend on each other, the equations have to be solved non-linearly in the second outermost coupling loop. When the converged solution 
of PBE is obtained, the corresponding $d_{32}$ field of the population is calculated and passed to the CFD part for the computation of the slip velocity in the next time step $t^{n+1}$, and the discretization of the equations in time is obtained according to the Crank-Nicolson method.
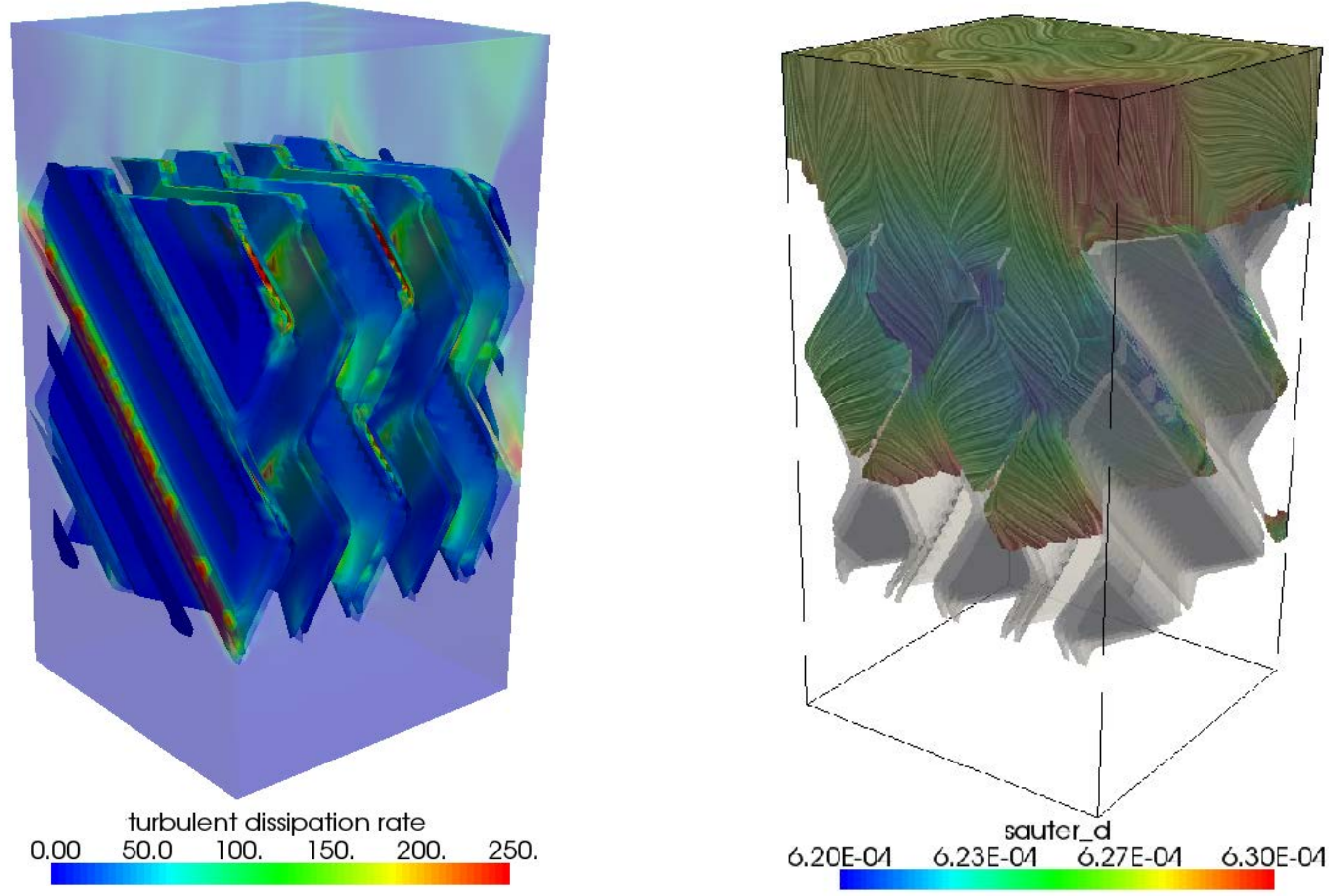

Figure 4: Values of turbulent dissipation rate (left) and droplet ensembles with $d_{32}$ (right).

\section{Results and discussions}

The developed computational tool was firstly used to simulate oil-in-water dispersions which is described with a one-way coupled PBE-CFD model. This model, its numerical treatment and the verified results are presented in the studies by Bayraktar et al. [21, 26]. Here we show a sample result from these studies, and later we present our results concerning the numerical simulation of Becker's experiment [18].

Table 1: Physical properties of the phases

\begin{tabular}{lcc}
\hline \hline Physical properties & Water & Oil \\
\hline$\rho\left(\mathrm{kg} \mathrm{m}^{-3}\right)$ & 1000 & 847 \\
$v\left(\mathrm{~kg} \mathrm{~m}^{-1} \mathrm{~s}^{-1}\right)$ & $1 \times 10^{-3}$ & $32 \times 10^{-3}$ \\
$\sigma\left(\mathrm{N} \mathrm{m}^{-1}\right)$ & $72 \times 10^{-3}$ & $21 \times 10^{-3}$ \\
$d_{32}(\mathrm{~m})$ & - & $1 \times 10^{-3}$ \\
\hline
\end{tabular}

Static mixers have a broad application field in various industrial processes. We study the Sulzer static mixer SMV ${ }^{\mathrm{TM}}$ both experimentally and numerically for various volume ratios 
of the phases and different volumetric flow-rates. The physical properties of the phases are given in Table 1 . This case is for the $10 \%$ holdup of the oil, the inflow is prescribed as a flat velocity profile with value $1 \mathrm{~m} / \mathrm{s}$, see Figure 5 . The turbulent dissipation rate field and droplet population with $d_{32}$ being in between $0.62 \mathrm{~mm}$ and $0.63 \mathrm{~mm}$ are visualized in Figure 4. Once we obtained the $d_{32}$ field, we calculate the average $d_{32}$ of the oil droplets at the outlet and compare them with the experimental results in Figure 5; for details of the computations we refer to Bayraktar et al. [26].
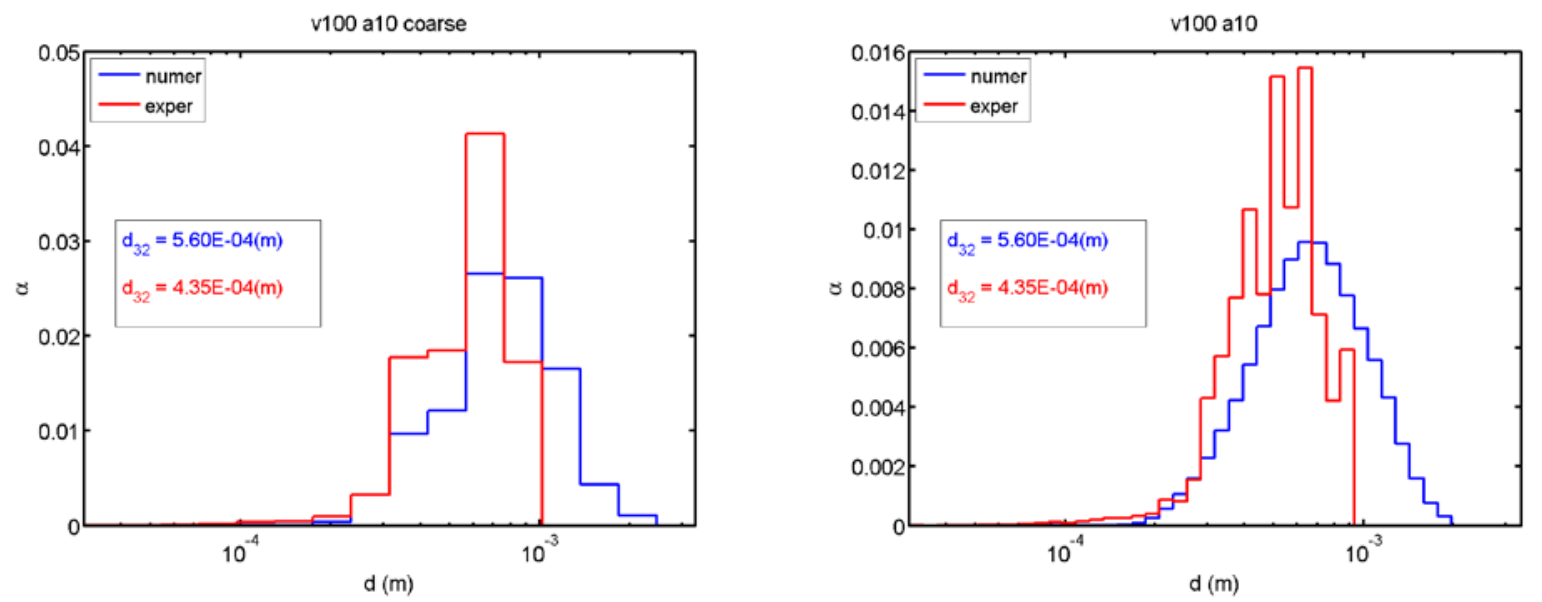

Figure 5: Comparison of experimental and numerical results on a coarse (15 classes, left) and fine grid (35 classes, right) in the internal coordinate.

The agreement in the numerical and the experimental results was the main motivation for us to extend our work with the two-way coupled CFD-PBE model and to study Becker's experiment. Becker et al. conducted experiments on the motion of bubble swarms in a partially aerated flat rectangular bubble column [18]. With the choice of a flat geometry the 3D flow field can be almost exactly characterized by 2D flow structures; so that, the observations of the flow field and measuring the relevant variables are easier and more accurate than doing it for 3D structures.

We simulate the bubble column within a computational domain $\Omega=(0,0.5) \times(0,1.5) \times(0,0.08) \mathrm{m}$ and on a Cartesian mesh of 6,912 hexahedral finite elements which result in $22848 \times 3$ degrees of freedom (dof) for velocity (Rannacher-Turek element, nonconforming trilinear $\tilde{Q}_{1}$ FE [27] ), 6912 dof for pressure (piecewise-constant $Q_{0} \mathrm{FE}$ ), $(2+11) \times 9125$ dof for $k-\varepsilon$ equations additional to PBE (conforming trilinear $Q_{1}$ FE). The circular gas sparger with $0.06 \mathrm{~m}$ diameter is located at the point $(0.15,0.00,0.04)$ and the prescribed volumetric flow rate is $1.6 \mathrm{l} / \mathrm{min}$. 
We prefer to start simulating breakage and coalescence on a developed flow field; hence, we obtain a developed flow field with a constant slip velocity $\left|u_{\text {slip }}\right|=0.2 \mathrm{~m} / \mathrm{s}$ in the opposite direction to gravity as Kuzmin et al. have done [2, 28]. After time $t=10 \mathrm{~s}$, we start to simulate the flow field with the population balance model coupled and the initial bubble size $d=1 \mathrm{~mm}$ which corresponds to the linearization parameter approximated by Kuzmin et al., $C_{W} \approx 5 \cdot 10^{4} \mathrm{~kg} \mathrm{~m}^{-3} \mathrm{~s}^{-1}$ is chosen.
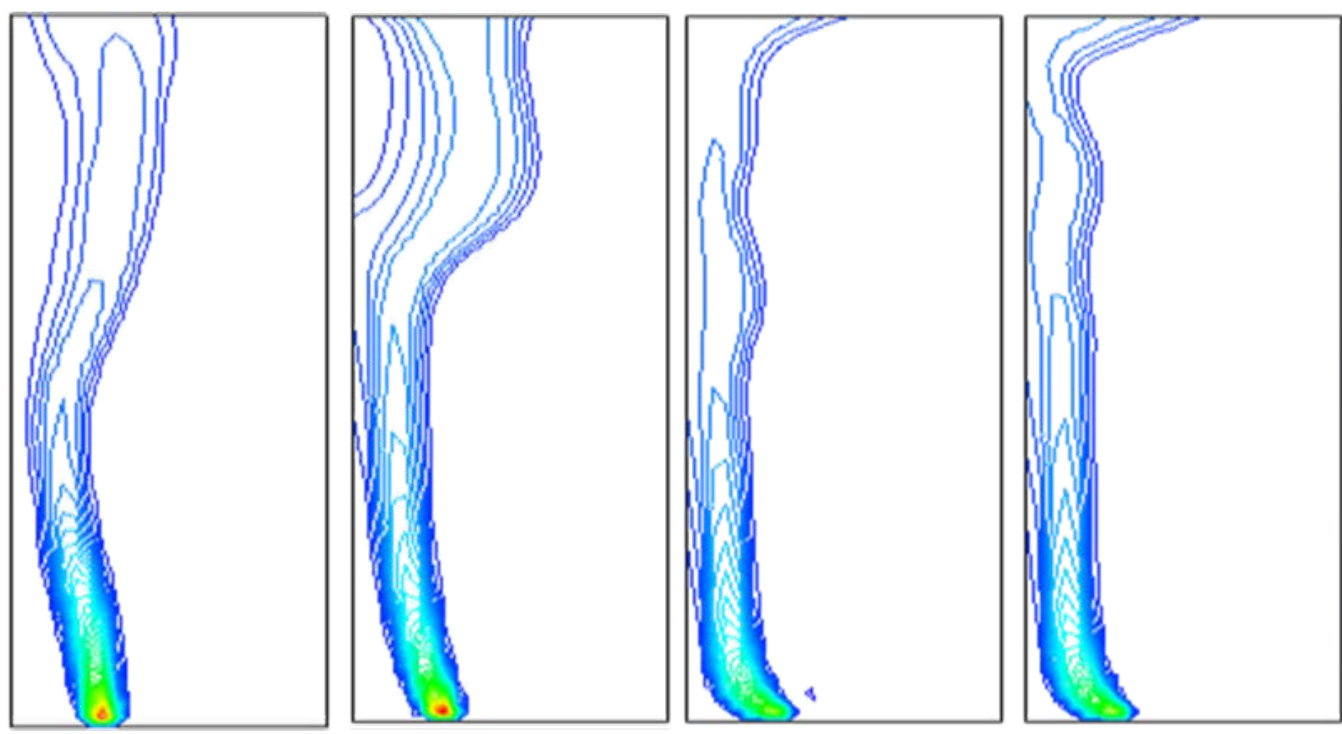

gholdup

$8.0 \mathrm{e}-02$
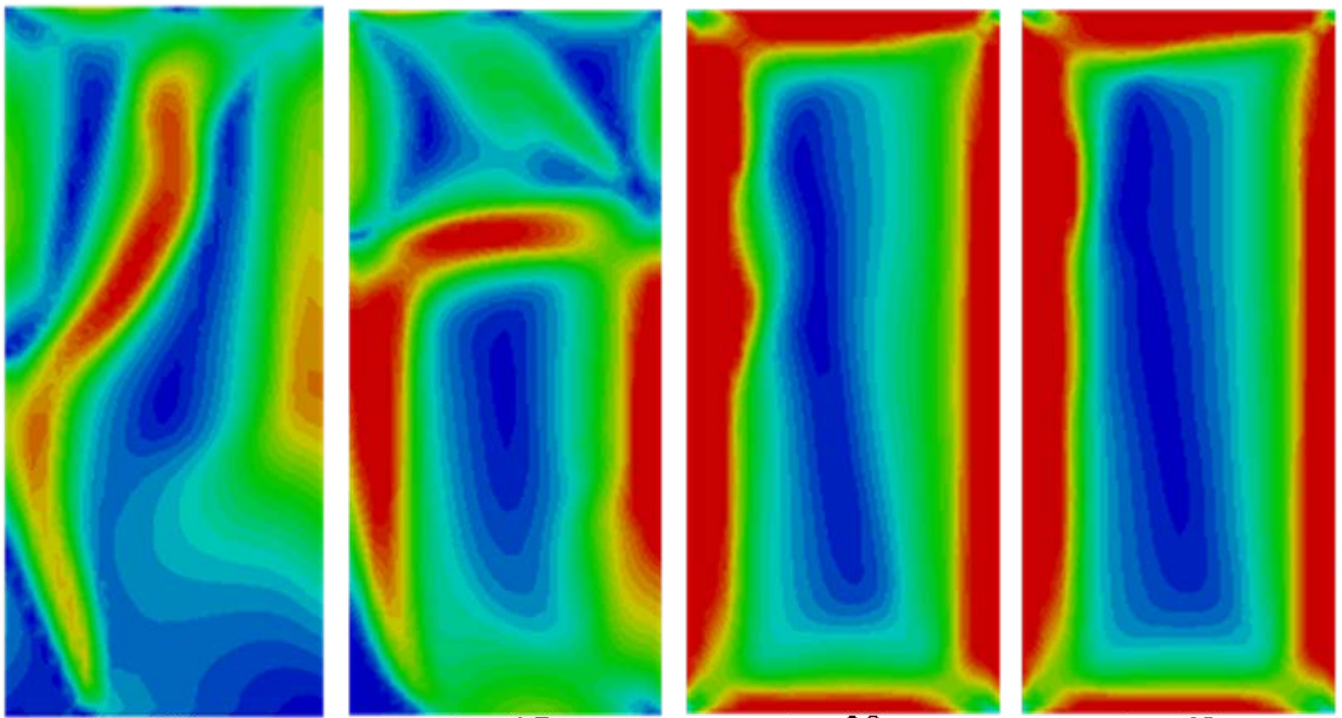

velocity

6.0e-02

4.0e-02

$2.0 \mathrm{e}-02$

$0.0 e+00$

$t=10 s$

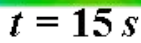

$t=30 s$

$t=60 s$

$-3.2 e-01$

$2.4 \mathrm{e}-01$

$1.6 e-01$

$8.0 \mathrm{e}-02$

$0.0 e+00$

Figure 6: Simulation results for the computational domain with 6,912 elements.

The simulation results are similar to the observations in the experiments, the meandering bubble swarm is numerically simulated until time $t=15 \mathrm{~s}$, see Figure 6 . In the experiments it is observed that the flow field does not tend to be steady in the long term. Our numerical simulations show that after the $15^{\text {th }}$ second the bubble swarms approaches to the 
near side wall at $t=30 \mathrm{~s}$; then, it leads to a quasi-steady flow field at $t=60 \mathrm{~s}$, see Figure 6 . Although, these results are more unsteady compared to the simulations without PBE, they are not yet satisfactory; however, we think that a computation that is performed on a finer spatial grid can yield to more agreeable results.

The slip velocity is one of the most influential variables on the flow field of the gas phase and challenging, and it requires that the gradient of the pressure is recovered. If the flow field is resolved on a finer grid, it can be recovered better; so, we homogeneously refined our hexahedral grid and obtained a grid of 55,296 hexahedral elements. The results which were obtained with this grid are able now to simulate the unsteady meandering bubble swarm even in the long run, see Figure 7.

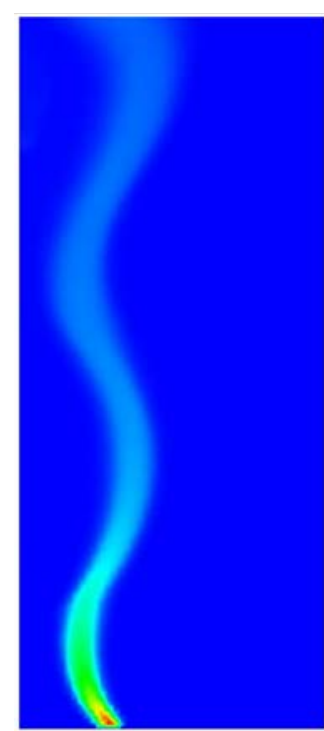

$t=30 s$

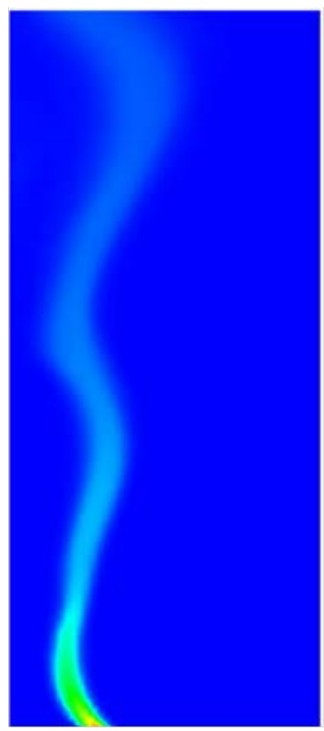

$t=35 s$

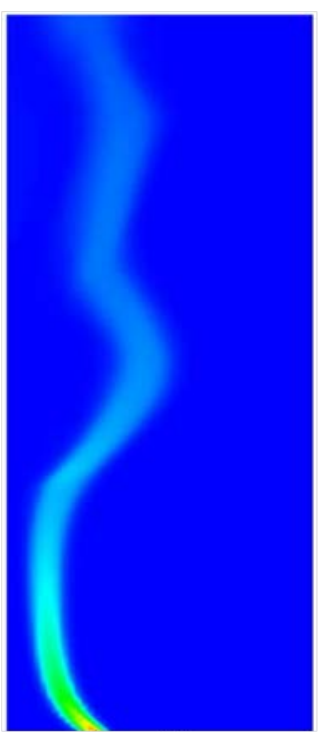

$t=40 s$

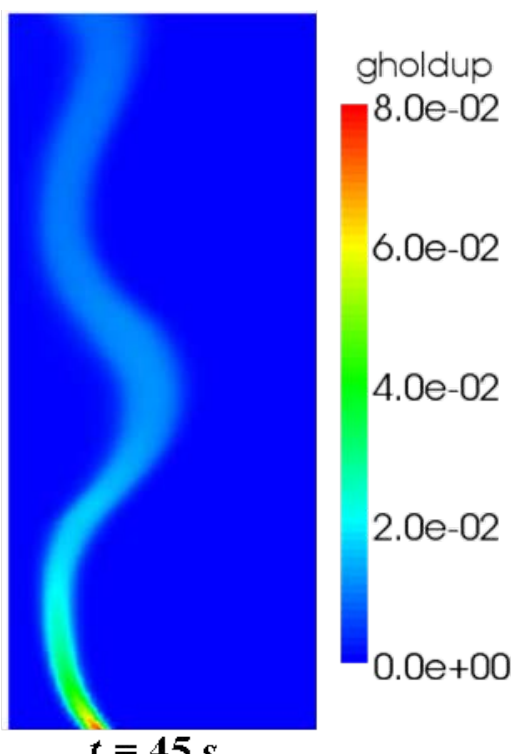

$t=45 s$

Figure 7: Simulation results with the fine computational grid.

We could numerically simulate the observed motion of bubble swarm in experiments; the numerical simulation predicts a qualitatively similar meandering behavior. A great challenge, to obtain a "non-diffusive" numerical scheme so that the dynamic behavior of bubbly flows can be numerically simulated for long-runs, is achieved within an efficient implementation of PBE coupled to CFD. In Table 2, the required computational time by different part of the PBE-CFD solver is presented for $60 \mathrm{~s}$ of the flow simulation which is performed on the coarse computational grid with 7 classes. The simulation is performed within a sequential computation on the AMD Opteron $250 \mathrm{CPU}$ with $2.4 \mathrm{GHz}$ frequency. The additional cost due to the incorporation of PBE is affordable and the required computational time remains on the same order with or without the PBE. The computation on the fine gird requires approximately 10 times larger computational time (approx. 1 week), which is at the 
edge of acceptable computational time for us. Therefore, although the Navier Stoke solver and the population balance solvers are efficiently parallelized within the domain decomposition method, a computation on a finer grid which would be good for the completeness of the results is currently not possible with our state-of-art turbulent-flow solver; and we leave it as a future task in our coming studies, particularly in combination with hardware-oriented techniques and GPU computing.

Table 2: Computational time required for the solution of different part of the model. (NS: Navier-Stoke Equ.; KE: Turbulence Equ.; $\alpha$ : Gas phase cont. Equ.; MP: Multiphase.)

\begin{tabular}{lcc}
\hline \hline Model & Time (s) & Time (\%) \\
\hline NS & 33500 & 55.4 \\
$\alpha$ & 4270 & 7.1 \\
KE & 13200 & 21.8 \\
PBE & 9475 & 15.7 \\
MP $($ PBE and $\alpha)$ & 13745 & 22.8 \\
\hline
\end{tabular}

\section{Conclusions}

PBE coupled with CFD simulations leads to agreeable results on a reasonably fine computational grid. The highly nonlinear coupled-model is very demanding with respect to computational efforts and, hence, requires very accurate and efficient numerical methods. The method of classes has shown to be a good choice, being able to describe the population with few classes, 7, that adds up to an affordable computational cost. The modified $k-\varepsilon$ models, e.g., Chien's Low-Reynolds number $k-\varepsilon$ turbulence model [21], should be tested since the resolved flow field was mildly turbulent. The spatial discretization is of great importance: both considering the employed finite element spaces and the mesh size. Positivity preserving linearization and high-order stabilization schemes are very crucial to avoid undershoots and overshoots. In this manner, a parallel implementation of a $Q_{2} / P_{1}$ solver for the incompressible multi-fluid model and a parallel high-order FEM-AFC solver with $Q_{1}$ elements for scalar transport equations (the PBE and the turbulence equations) is very promising to simulate bubbly flows. Moreover, simulations will be more comprehensive if the motion of the water surface is resolved with a surface tracking or surface capturing method, e.g., level set method [29]. In case of highly turbulent bubbly flows where the dispersion can exhibit a wide distribution in the internal coordinate, the method of classes might be less efficient; then, other methods, e.g. DQMOM, QMOM, can be employed. 


\section{Acknowledgments}

Dr. Dimitri Kuzmin's studies in the field of bubbly flows are highly acknowledged. The authors like to thank Sulzer Innotec, Sulzer Markets and Technology AG for supporting Evren Bayraktar with a doctoral scholarship. The support by the LiDOng team at the ITMC at TU Dortmund is gratefully acknowledged.

\section{Symbols used}

$\begin{array}{ll}C & \text { turbulence model constants (with subscript) } \\ d & \text { diameter of bubbles } \\ f & \text { number density probability function } \\ f_{B V} & \text { bubble/droplet volume fraction } \\ f_{1}, f_{2}, f_{\mu} & \text { damping functions } \\ g & \text { gravitational acceleration } \\ k & \text { turbulent kinetic energy } \\ N & \text { number of classes } \\ P & \text { production term due to shear, } \\ P & \text { pressure } \\ Q & \text { discretization factor } \\ R & \text { kernel } \\ S & \text { production term due to BIT } \\ u & \text { velocity } \\ u^{\prime} & \text { fluctuating velocities }\end{array}$

Greek letters

A

$\Delta v$

E

$\rho$

$v_{T}$

$\sigma$

$\sigma_{T}$

v void fraction

class width

turbulent dissipation rate

density

eddy viscosity

turbulence model constants (with subscript)

turbulent Schmidt number

volume 


\section{Subscripts}

D drag

G

Gas

K

turbulent kinetic energy related

$i, j, k$

class indices (in the context of PBE)

$L$

liquid

E

turbulent dissipation rate related

W

linearization coefficient

$L$

liquid

T

turbulent

Superscripts

$B, C$

Breakage, coalescence

$L, U$

lower, upper limit of classes

$\sim$

Daughter bubble/droplet

$+,-$ source, sink 


\section{References}

[1] M. Millies, D. Mewes, Chem. Eng. Process., 1999, 38, 307-319. DOI: 10.1016/S02552701(99)00022-7.

[2] D. Kuzmin, S. Turek, H, Haario, 2005, Ergebnisberichte des Instituts für Angewandte Mathematik, Nummer 298, Fakultät für Mathematik, TU Dortmund.

[3] R. McGraw, Aerosol Sci. Technol., 1997, 27, 255-265. DOI: 10.1080/02786829708965471.

[4] R. McGraw, Wright D. L., J. Aerosol Sci., 2003, 34, 189-209. DOI: 10.1016/S00218502(02)00157-X.

[5] R. Fan, D. L. Marchisio, R. O. Fox, Powder Techno., 2004, 139, 7-20. DOI: 10.1016/j.powtec.2003.10.005.

[6] S. Bove, PhD Thesis, Aalborg University, Denmark, 2005.

[7] S. Kumar, D. Ramkrishna, Chem. Eng. Sci., 1996, 51 (8), 1311-1332. DOI: 10.1016/0009-2509(96)88489-2.

[8] S. Kumar, D. Ramkrishna, Chem. Eng. Sci., 1996, 51 (8), 1333-1342. DOI: 10.1016/0009-2509(95)00355-X.

[9] A. Sokolichin, G. Eigenberger, A. Lapin, A. Lübert, Chem. Eng. Sci., 1997, 52 (5), 611-626. DOI: 10.1016/S0009-2509(96)00425-3.

[10] H. Rusche, PhD Thesis, University of London, England, 2002.

[11] M. Laakkonen, P. Moilanen, V. Alopaeus, J. Aittamaa, Chem. Eng. Sci., 2007, 62 (5), 721-740. DOI: 10.1016/j.ces.2006.10.006.

[12] H. A. Jakobsen, H. Lindborg, C. A. Dorao, Ind. Eng. Chem. Res., 2005, 44, 5107-5151. DOI: $10.1021 / \mathrm{ie} 049447 \mathrm{x}$.

[13] R. Bannari, F. Kerdouss, B. Selma, A. Bannari, P. Proulx, Comput. Chem. Eng., 2008, 32 (12). DOI: 10.1016/j.compchemeng.2008.05.016.

[14] B. Selma, R. Bannari, P. Proulx, Chem. Eng. Sci., 2010, 65 (6), 1925-1941. DOI: 10.1016/j.ces.2009.11.018.

[15] R. O. Fox, F. Laurent, M., Massot, J. Comput. Phys., 2008, 227 (6), 3058-3088. DOI: 10.1016/j.jcp.2007.10.028.

[16] A. Vi, F. Laurent, M. Massot, Size-velocity correlations in high order moment methods for polydisperse evaporating sprays: modelling and numerical issues, 2011. Available at http://hal.archives-ouvertes.fr .

[17] A. Sokolichin, G. Eigenberger, A. Lapin, AIChE J., 2004, 50, 24-45. DOI: 10.1002/aic.10003. 
[18] S. Becker, A. Sokolichin, G. Eigenberger, Chem. Eng. Sci., 1994, 49, 5747-5762. DOI: 10.1016/0009-2509(94)00290-8.

[19] A. Sokolichin, Habilitationsschrift, Universität Stuttgart, Stuttgart, 2004.

[20] D. Pfleger, S. Gomes, N. Gilbert, H. G. Wagner, Chem. Eng. Sci., 1999, 54 (21), 5091-5099. DOI: 10.1016/S0009-2509(99)00261-4.

[21] E. Bayraktar, O. Mierka, F. Platte, D. Kuzmin, S. Turek, Comput. Chem. Eng., 2011, 35 (11), 2204-2217. DOI: 10.1016/j.compchemeng.2011.04.001.

[22] D. Kuzmin, M. Möller, in Flux-Corrected Transport: Principles, Algorithms, and Applications (Eds: D. Kuzmin, R. Löhner, S. Turek), Springer, Berlin Heidelberg, 2005, 155-206.

[23] S. Turek, D. Kuzmin, in Flux-Corrected Transport: Principles, Algorithms, and Applications (Eds: D. Kuzmin, R. Löhner, S. Turek), Springer, Berlin Heidelberg, 2005, 251-296.

[24] P. Chen, J. Sanyal, M. P. Duduković, Chem. Eng. Sci., 2005 (4), 60, 1085-1101. DOI: 10.1016/j.ces.2004.09.070.

[25] F. Lehr, M. Millies, D.Mewes, AIChE J., 2002, 48 (11), 2426-2442. DOI:10.1002/aic.690481103.

[26] E. Bayraktar, R. Münster, O. Mierka, S. Turek, in Proc. of the 6th Int. Berlin Workshop on Transport Phenomena with Moving Boundaries (Eds: F. P. Schindler, M. Kraume), VDI, Berlin, 2011.

[27] R. Rannacher, S. Turek, Numer. Meth. PDEs, 1992, 8 (2), 97-11.

[28] D. Kuzmin, S. Turek, in Proc. of the 3rd Int. Symposium on Two-Phase Flow Modeling and Experimentation, Pisa, 2004.

[29] S. Turek, O. Mierka, S. Hysing, D. Kuzmin, in Numerical methods for differential equations, optimization, and technological problems (Eds: S. Repin, T. Tiihonen, T. Tuovinen), Springer, Berlin Heidelberg, 2012.

[30] D. Kuzmin, S. Turek, in Conjugate gradient algorithms and finite element methods (Eds: M.Krizek et al.), Springer, Berlin Heidelberg, 2004.

[31] D. Kuzmin, R. Löhner, S. Turek, in Flux-corrected transport, (Eds: D. Kuzmin, R. Löhner, S. Turek), Springer, Berlin Heidelberg, 2005. 